\title{
Attenuation of renal ischemia/reperfusion injury by oleanolic acid preconditioning via its antioxidant, anti-inflammatory, and anti-apoptotic activities
}

\author{
CHENGMEI LONG ${ }^{1,2}$, JINRAN YANG $^{2}$, HUA YANG $^{2}$, XINCHANG LI $^{2}$ and GONGXIAN WANG ${ }^{3}$ \\ ${ }^{1}$ Graduate Faculty, Medical College of Nanchang University; ${ }^{2}$ Department of Organ Transplantation, \\ Jiangxi Provincial People's Hospital; ${ }^{3}$ Department of Urology, The First Affiliated Hospital of Nanchang University, \\ Nanchang, Jiangxi 330006, P.R. China
}

Received March 16, 2015; Accepted January 20, 2016

DOI: $10.3892 / \mathrm{mmr} .2016 .5128$

\begin{abstract}
Ischemia/reperfusion (I/R)-associated acute kidney injury is a major clinical problem in both native and transplanted kidneys. Renal I/R, and subsequent renal injury, may be attributed to oxidative stress, inflammation, and apoptosis. Oleanolic acid (OA) is a natural product, which possesses antioxidant, anti-inflammatory, and anti-apoptotic activities. The present study aimed to examine the effects of OA preconditioning on renal $\mathrm{I} / \mathrm{R}$ and the possible underlying mechanisms. In a renal I/R model, rats were administered OA $(12.5,25$ and $50 \mathrm{mg} / \mathrm{kg}$ ) for 15 consecutive days prior to bilateral renal I/R induction. Serum samples and kidneys were then collected and stored for subsequent determination. The results of the present study demonstrated that OA significantly and dose-dependently attenuated I/R-induced renal damage. OA prevented renal I/R injury, as evidenced by decreased levels of blood urea nitrogen, creatinine, kidney injury molecule-1 and lactate dehydrogenase. In addition, OA defended against oxidative stress, as reflected by decreased levels of methane dicarboxylic aldehyde, increased activities of superoxide dismutase, catalase and glutathione peroxidase, and increased glutathione (GSH) levels. Levels of proinflammatory cytokines, interferon- $\gamma$, interleukin (IL)-6) and myeloperoxidase, were also reduced by OA, whereas the anti-inflammatory cytokine IL-10 was increased. Furthermore, OA prevented I/R-induced apoptotic cell death, and prevented decreases in the mRNA expression levels of nuclear factor erythroid 2-related factor 2 (Nrf2) and $\gamma$-glutamylcysteine ligase (GCLc). Conversely, buthionine sulphoximine attenuated the protective effects of OA on renal $\mathrm{I} / \mathrm{R}$ injury. These results indicated that OA preconditioning
\end{abstract}

Correspondence to: Miss. Gongxian Wang, Department of Urology, The First Affiliated Hospital of Nanchang University, 17 Yong Wai Street, Nanchang, Jiangxi 330006, P.R. China E-mail: gongxian_wang15@163.com

Key words: renal ischemia/reperfusion, oleanolic acid, antioxidant, anti-inflammatory, anti-apoptotic may prevent I/R-induced renal damage via antioxidant, anti-inflammatory, and anti-apoptotic activities. Stabilization of Nrf2/GCLc signaling and subsequent maintenance of the GSH pool is critical for the protective effects of OA against renal I/R injury. The present study reported a novel therapeutic strategy for the treatment of renal I/R injury.

\section{Introduction}

Ischemia/reperfusion (I/R) injury, which is a common pathophysiological condition in various tissues, occurs when blood supply returns to the tissue following a period of ischemia. Renal I/R damage is a major cause of acute kidney injury after partial nephrectomy or renal transplantation $(1,2)$, which is associated with severe morbidity and mortality (3). Effective interventions or treatments are required for the attenuation of renal I/R injury.

Renal I/R injury is a complex process that involves oxidative stress (4), inflammation (5), and apoptosis (6,7). Increased levels of reactive oxygen species (ROS) and/or decreased antioxidant enzyme activity are key events following renal I/R, which may activate subsequent damage (8). Under renal I/R conditions, various proinflammatory factors are elevated (9), and activation of the caspase pathway and subsequent apoptotic cell death results in renal cytotoxicity (10). These processes are intertwined, leading to a vicious circle of damage (11). Antioxidant, anti-inflammatory, and anti-apoptotic effects have been reported to ameliorate renal $\mathrm{I} / \mathrm{R}$ injury to a certain degree (9-13).

Oleanolic acid (OA) is a natural triterpenoid and an aglycone of several saponins. OA is present in various food products, including vegetable oils, and is a main constituent of the leaves and roots of Olea europaea, Viscum album L., Aralia chinensis L., and $>120$ other plant species (14). In the past three decades, OA has been used in Chinese medicine for the treatment of liver disorders, including viral hepatitis. In vitro and in vivo studies have demonstrated that $\mathrm{OA}$ possesses potent antioxidant activities $(15,16)$, and anti-inflammatory effects (17), which contribute toward its potential protective effects. However, whether OA exerts protective effects against renal I/R injury, and the possible underlying mechanism, 
remain unclear. The present study aimed to evaluate the renal protective effects of OA against I/R injury, and to examine the potential underlying molecular mechanism. The results indicated that OA preconditioning effectively prevented renal I/R injury via antioxidant, anti-inflammatory, and anti-apoptotic activities. Activation of nuclear factor erythroid 2-related factor 2 (Nrf2)/ $\gamma$-glutamylcysteine ligase (GCLc) signaling, and upregulation of glutathione (GSH) may have an important role in the preventive effects of OA against renal I/R injury.

\section{Materials and methods}

Animals and treatment. All animal experimental protocols were performed in accordance with the standards established by the Animal Care and Use Committee of the Medical College of Nanchang University (Nanchang, China). The present study was approved by the ethics committee of the Medical College of Nanjing University. Male adult Wistar rats (weight, 180-200 g; age, 6-8 weeks) were obtained from the Animal Center of the Medical College of Nanchang University and housed in stainless steel cages with ad libitum access to food and water. Two protocols were subsequently conducted, the OA and buthionine sulphoximine (BSO) used were obtained from Sigma-Aldrich (St. Louis, MO, USA). Protocol 1: A total of 30 rats were randomly divided into five groups: The sham group, which received sham surgery; the $\mathrm{I} / \mathrm{R}$ group; the $12.5 \mathrm{mg} / \mathrm{kg} \mathrm{OA}+\mathrm{I} / \mathrm{R}$ group, which received $12.5 \mathrm{mg} / \mathrm{kg}$ OA for 15 consecutive days prior to the operation; the $25 \mathrm{mg} / \mathrm{kg} \mathrm{OA}+\mathrm{I} / \mathrm{R}$ group, which received $25 \mathrm{mg} / \mathrm{kg}$ $\mathrm{OA}$ for 15 consecutive days prior to the operation; and the $50 \mathrm{mg} / \mathrm{kg} \mathrm{OA}+\mathrm{I} / \mathrm{R}$ group, which received $50 \mathrm{mg} / \mathrm{kg} \mathrm{OA}$ for 15 consecutive days prior to the operation. Protocol 2: A total of 24 rats were randomly divided into four groups: The sham group, which received sham surgery; the I/R group; the $50 \mathrm{mg} / \mathrm{kg} \mathrm{OA}+\mathrm{I} / \mathrm{R}$ group, which received $50 \mathrm{mg} / \mathrm{kg} \mathrm{OA}$ for 15 consecutive days prior to the operation); and the $50 \mathrm{mg} / \mathrm{kg}$ $\mathrm{OA}+\mathrm{BSO}+\mathrm{I} / \mathrm{R}$ group, which received $50 \mathrm{mg} / \mathrm{kg} \mathrm{OA}$ and $10 \mathrm{mg} / \mathrm{kg}$ BSO for 15 consecutive days prior to the operation. Rats were intraperitoneally injected with OA in sterile saline containing $2 \%$ Tween 80 .

Model of renal I/R injury. Rats were fasted overnight, and were then anaesthetized with pentobarbital sodium $(50 \mathrm{mg} / \mathrm{kg}$; i.p.; Sigma-Aldrich) prior to the operation. Renal IR injury was established as previously described $(11,18)$. Briefly, a midline incision was made, and the kidneys and renal pedicles containing the artery, vein and nerve of each kidney were exposed. The bilateral renal pedicle was exposed and clamped with a vascular clamp for $45 \mathrm{~min}$, in order to induce ischemia. The clamps were then removed to allow reperfusion for $6 \mathrm{~h}$. Pale coloring was considered to indicate ischemia of the kidneys, which returned to red post-reperfusion. In the control rats, the same surgical procedure was conducted without the clamping.

Following reperfusion, the rats were anaesthetized with $50 \mathrm{mg} / \mathrm{kg}$ pentobarbital sodium and decapitated, and blood samples were collected for serum separation, and the kidneys were removed. Blood samples were stored overnight at $4^{\circ} \mathrm{C}$ and centrifuged at $664 \mathrm{x} \mathrm{g}$ for $10 \mathrm{~min}$ to separate the serum. Serum and kidney samples were stored at $-70^{\circ} \mathrm{C}$ until further analysis.
Evaluation of renal function. The serum concentrations of blood urea nitrogen (BUN) and creatinine $(\mathrm{Cr})$ were detected to assess renal function using a QuantiChromTM Urea assay kit and Creatinine Colorimetric assay kit (Bioassay Systems LLC, Hayward, CA, USA), according to the manufacturer's protocols. Kidney injury molecule-1 (KIM-1) content was examined using a KIM-1 enzyme-linked immunosorbent assay (ELISA) kit (USCN Life Science Inc., Wuhan, China), according to the manufacturer's protocol. Serum lactate dehydrogenase (LDH) activity was evaluated using a LDH Colorimetric assay kit (BioVision Inc., Milpitas, CA, USA) according to the manufacturer's protocols.

Evaluation of antioxidant defense. Renal tissues were homogenized for evaluation of antioxidant defense. Methane dicarboxylic aldehyde (MDA) levels were determined as described previously (19), using the colorimetric absorption of the 2-thiobarbituric acid-MDA chromophore for detection of lipid peroxidation. This was conducted using an MDA assay kit (Nanjing Jiancheng Bioengineering Research Institute, Nanjing, China). Renal superoxide dismutase (SOD) activity was assayed using a Total SOD assay kit (Nanjing Jiancheng Bioengineering Research Institute) based on reduction of nitroblue tetrazolium (NBT) by $\mathrm{O}_{2}{ }^{--}$produced by hydroxylamine hydrochloride autoxidation, as described previously (20). One unit of SOD was defined as the amount of protein that caused a $50 \%$ inhibition in the rate of NBT reduction. A microplate reader was used to determine absorbance in the MDA and SOD assays (Infinite 200; Tecan Group, Ltd., Männedorf, Switzerland). Renal activity of catalase (CAT) was determined using a CAT assay kit (Nanjing Jiancheng Bioengineering Research Institute) according to the manufacturer's protocols. One unit of CAT was defined as the amount required to decompose $1 \mu \mathrm{mol} \mathrm{H}_{2} \mathrm{O}_{2}$ per second. Glutathione (GSH) and glutathione peroxidase (GPx) activity was assayed using a GSH Fluorometric assay kit and GPx assay kit (BioVision Inc.), according to the manufacturer's protocols.

Determination of inflammation. Levels of interferon- $\gamma$ (IFN- $\gamma$ ), interleukin (IL)-6 and IL-10 were detected in homogenized renal tissue using specific CLIA kit for IL-6 and CLIA kit for IL-10 (USCN Life Science Inc. and Eagle Biosciences, Inc., Nashua, NH, USA, respectively). Renal myeloperoxidase (MPO) levels were also examined using a Myeloperoxidase ELISA kit (ALPCO, Salem, NH, USA). These assays were conducted according to the manufacturers' protocols.

Determination of apoptosis. Renal tissue was cut into 10-mm sections and fixed in paraformaldehyde (Sigma-Aldrich) for use in determination of apoptosis. A terminal deoxynucleotidyl transferase-mediated dUTP nick-end labeling (TUNEL) assay was conducted to evaluate the rate of apoptosis in renal sections using an In Situ Cell Death Detection kit (Roche Diagnostics, Basel, Switzerland) according to the manufacturer's protocols. The number of apoptotic cells was counted in 10 random fields using a BX51TF microscope at magnification x200 (Olympus Corporation, Tokyo, Japan). Renal caspase 3 content was detected in homogenized renal tissue using an ELISA kit for Caspase 3 (USCN Life Science Inc.) according to the manufacturer's protocol. 

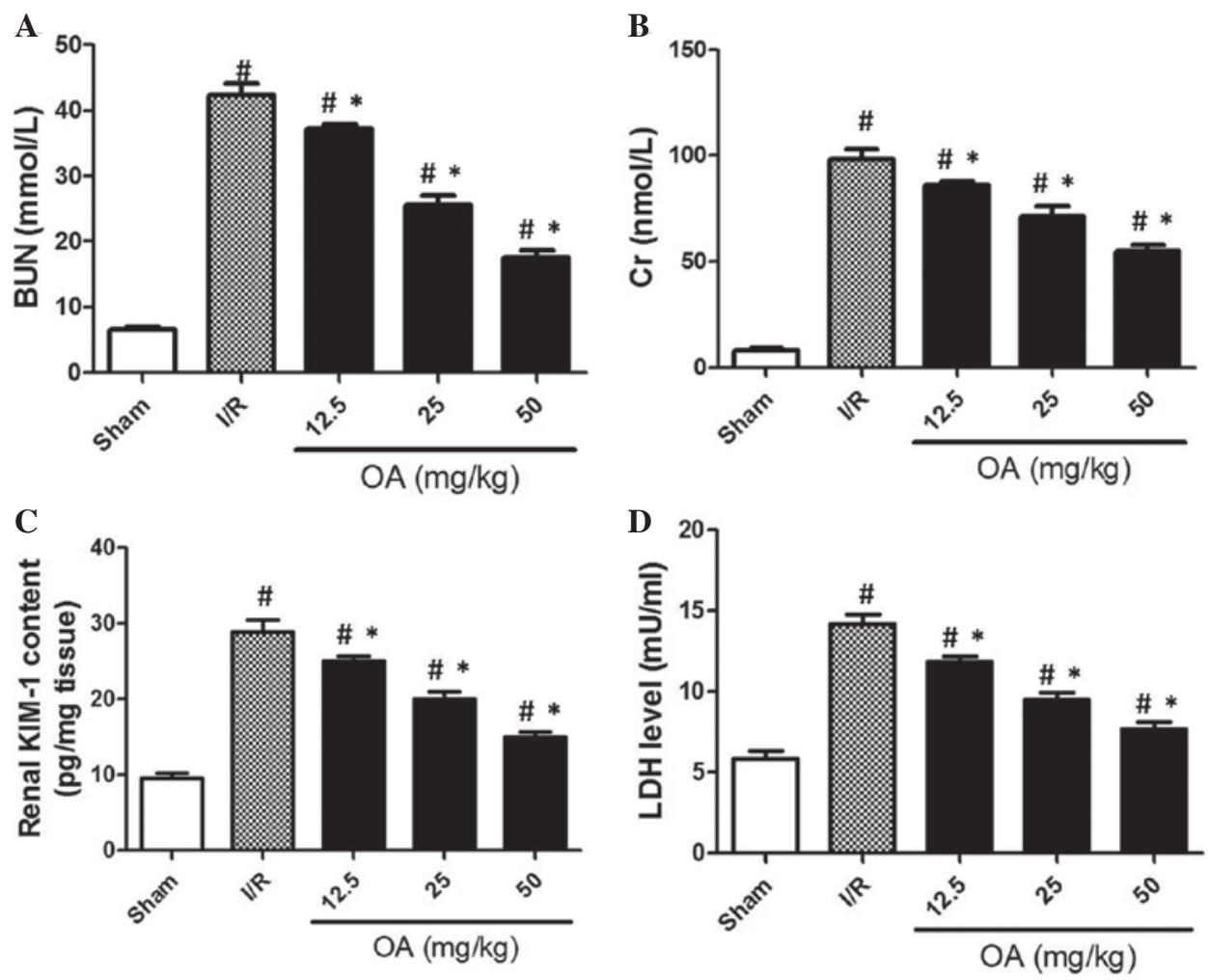

Figure 1. Effects of oleanolic acid (OA) preconditioning on renal ischemia/reperfusion (I/R) injury. Rats were pre-injected with OA for 15 consecutive days prior to I/R operation. Subsequently, serum levels of (A) blood urea nitrogen (BUN) and (B) creatinine (Cr), and (C) renal kidney injury molecule-1 (KIM-1) and (D) serum lactate dehydrogenase ( $\mathrm{LDH})$ levels were measured using commercial kits. Data are presented as the mean \pm standard error. ${ }^{\#} \mathrm{P}<0.05$, compared with the sham control group; ${ }^{*} \mathrm{P}<0.05$, compared with the I/R group.

Reverse transcription-quantitative polymerase chain reaction (RT-qPCR). Total RNA was purified from renal tissue using RNeasy Plus kit (Qiagen, Inc., Valencia, CA, USA), according to the manufacturer's protocol. Total RNA $(0.5 \mu \mathrm{g})$ was reverse transcribed using the Fast $\mathrm{SYBR}^{\circledR}$ Green Master Mix according to the manufacturer's instructions (Takara Bio, Inc., Otsu, Japan). The RT-qPCR assay was performed in triplicate in reaction volumes of $20 \mu \mathrm{l}$ containing $10 \mu \mathrm{l}$ Fast SYBR Green Master Mix (Thermo Fisher Scientific, Inc., Waltham, MA, USA), $1 \mu \mathrm{l}$ of sense and antisense primers $(0.5 \mu \mathrm{M}$; Augct DNA-Syn Biotechnology Co., Ltd., Beijing, China), $1 \mu$ cDNA (50 ng/20 $\mu \mathrm{l}$ ) and $7 \mu \mathrm{l}$ double distilled water. The reactions were performed in a CFX96 Real-Time PCR system (Bio-Rad Laboratories, Inc., Hercules, CA, USA). The cycling conditions used were: Initial activation for $5 \mathrm{~min}$ at $95^{\circ} \mathrm{C}$; and 40 cycles of denaturation for $5 \mathrm{sec}$ at $95^{\circ} \mathrm{C}$ and $10 \mathrm{sec}$ of combined annealing and extension at the individual primer temperature between 55 and $62.5^{\circ} \mathrm{C}$. The relative amount of each gene was normalized to $\beta$-actin. The primer sequences used were as follows: $\beta$-actin, sense 5'-AGATCCTGACCGAGCGTGGC-3', antisense 5'-CCAGGGAGGAAGAGGATGCG-3'; Nrf2, sense 5'-TCAGCTACTCCCAGGTTGCCCA-3', antisense 5'-GGC AAGCGACTCATGGTCATCTAC-3'; and GCLc, sense: 5'-AACACAGACCCAACCCAGAG-3' and antisense 5'-CCG CATCTTCTGGAAATGTT-3'. Gene expression levels were analyzed using the $2^{-\Delta \Delta \mathrm{Cq}}$ method (21).

Statistical analysis. Statistical analysis of the data was performed using GraphPad software, version 5.0 (GraphPad
Software, Inc., La Jolla, CA, USA). The results are presented as the mean \pm standard error. Statistical significance of the differences between the groups was analyzed using one-way analysis of variance followed by Newman-Keuls multiple comparison post-hoc test. $\mathrm{P}<0.05$ was considered to indicate a statistically significant difference.

\section{Results}

$O A$ preconditioning prevents renal $I / R$ injury. The present study consisted of two protocols. In protocol 1, the dose-dependent effects of 12.5-50 mg/ $\mathrm{kg}$ OA were detected on the renal function of $\mathrm{I} / \mathrm{R}$ rats. In protocol $2, \mathrm{BSO}$ was used to examine the role of GSH regulation in OA-induced prevention of renal $I / R$ injury. In order to evaluate renal function, several related parameters were determined. In the case of renal insufficiency, BUN and $\mathrm{Cr}$ are retained in the blood, and their excretion by the kidneys is impaired. As shown in Fig. $1 \mathrm{~A}$ and B, BUN and Cr levels were significantly increased $\sim 5$-fold in the I/R group compared with in the sham control group $(\mathrm{P}=0.0008)$. Conversely, OA preconditioning dose-dependently prevented the increased levels of BUN and $\mathrm{Cr}$ in the I/R rats (Fig. $1 \mathrm{~A}$ and $\mathrm{B} ; \mathrm{P}=0.0001$ ). $\mathrm{KIM}-1$ is a novel biomarker for human renal proximal tubule injury (22). The present study demonstrated that in I/R rats, KIM-1 content was increased $\sim 3$-fold compared with in the sham control group (Fig. 1C; $\mathrm{P}=0.0004$ ). OA preconditioning significantly reduced KIM-1 content in I/R rats in a dose-dependent manner (Fig. 1C). Following tissue injury, LDH may be released into 

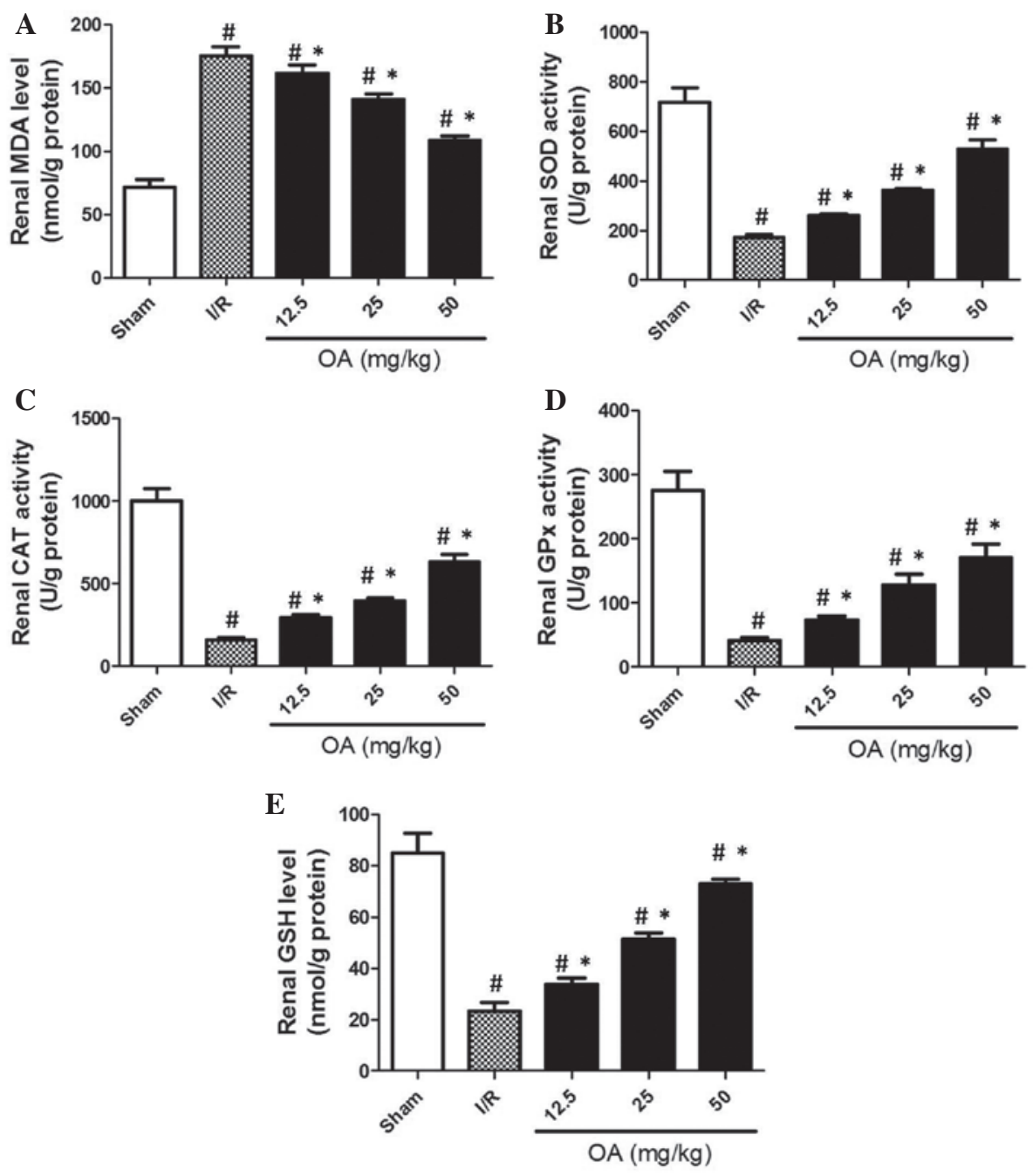

Figure 2. Effects of oleanolic acid (OA) preconditioning on antioxidant defense in a rat model of ischemia/reperfusion (I/R). Rats were pre-injected with OA for 15 consecutive days prior to I/R operation. Subsequently, renal (A) methane dicarboxylic aldehyde (MDA) levels, (B) superoxide dismutase (SOD), (C) catalase (CAT), (D) glutathione peroxidase (GPx) activities, and (E) glutathione (GSH) content were determined. Data are presented as the mean \pm standard error. ${ }^{\text {"}} \mathrm{P}<0.05$, compared with the sham control group; $\mathrm{P}<0.05$, compared with the I/R group.

the bloodstream. In the circulation of $\mathrm{I} / \mathrm{R}$ rats, $\mathrm{LDH}$ release was markedly increased. Conversely, OA preconditioning markedly prevented this increase in LDH release (Fig. 1D; $\mathrm{P}=0.002)$. These results indicate that $\mathrm{OA}$ preconditioning may effectively prevent I/R-induced renal injury in rats. Since $50 \mathrm{mg} / \mathrm{kg}$ exhibited the most significant protective effect on these indices, this dose was used in protocol 2.

Antioxidative effects of $\mathrm{OA}$ preconditioning are associated with the prevention of renal I/R injury. To evaluate antioxidant injury in I/R rats, several key markers of antioxidant activity were detected. MDA is an important end-point marker of lipid peroxidation. As shown in Fig. 2A, MDA levels were increased $>2$-fold in the kidneys of $\mathrm{I} / \mathrm{R}$ rats compared with in the sham control group. OA preconditioning markedly prevented the increased levels of MDA in a dose-dependent manner (Fig. 2A; $\mathrm{P}=0.0009$ ). SOD is an important antioxidant enzyme, which catalyzes the dismutation of superoxide to oxygen and hydrogen peroxide. In turn, hydrogen peroxide may be broken down by CAT. The results of the present study demonstrated that in I/R rats, the serum activities of SOD and
CAT were markedly decreased (Fig. 2B and C). As expected, OA preconditioning prevented I/R-induced decreased renal activity of SOD and CAT (Fig. 2B and C; P=0.0003 and $\mathrm{P}=0.001$, respectively). Furthermore, GPx is a key antioxidant enzyme that eliminates organic peroxide using GSH as a reductive equivalent. As shown in Fig. 2D, I/R induced a significant decrease in GPx activity, which was prevented by OA preconditioning $(\mathrm{P}=0.003)$. Furthermore, $\mathrm{GSH}$ is a major cellular antioxidant in cells, which is able to detoxify various oxidative products directly, or under the catalyzation of GPx or glutathione S-transferase. The results demonstrated that $\mathrm{I} / \mathrm{R}$ resulted in a marked reduction in GSH renal content (Fig. 2E; $\mathrm{P}=0.0045$ ). OA preconditioning markedly prevented the I/R-induced decreased renal content of GSH. These results suggest that $\mathrm{I} / \mathrm{R}$ induced oxidative stress in the rat kidneys, and the preventive effects of OA may be due to its antioxidant activity.

Anti-inflammatory effects of OA preconditioning are associated with the prevention of renal I/R injury. The process of inflammation is associated with $I / R$-induced injury in various 

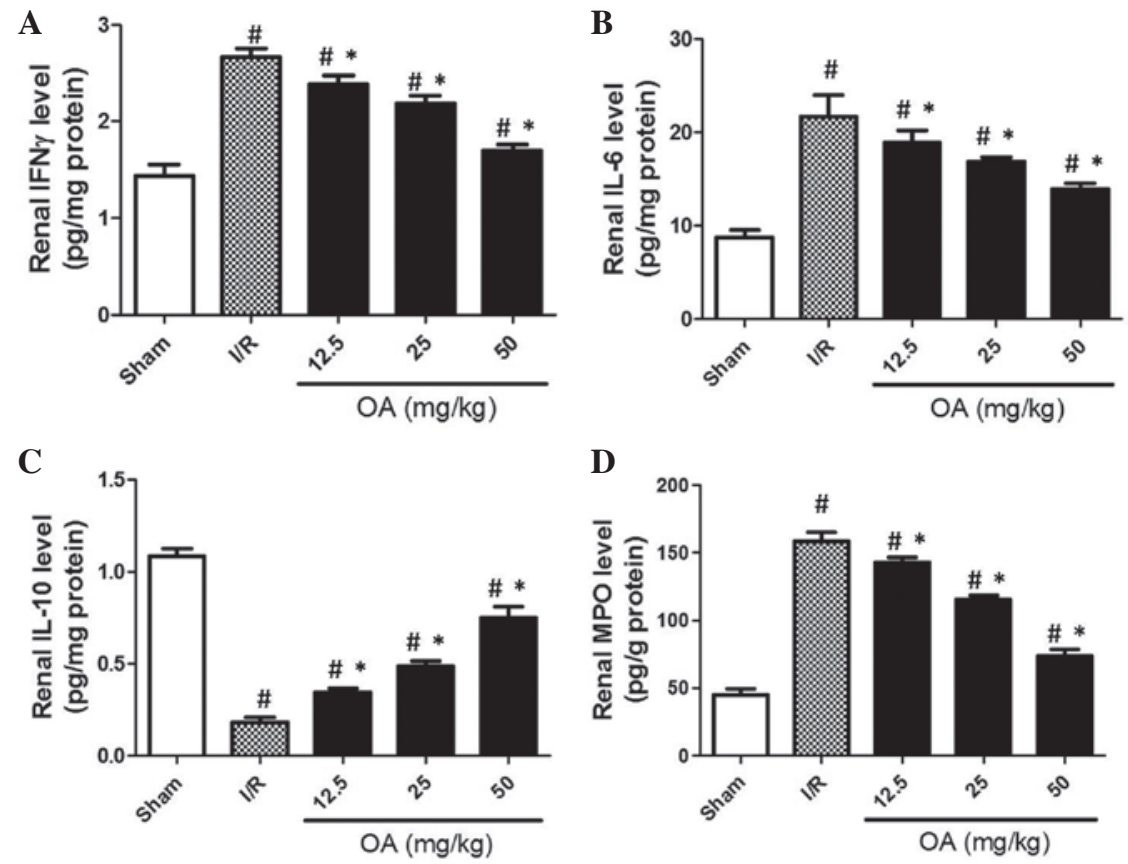

Figure 3. Effects of oleanolic acid (OA) preconditioning on inflammation in a rat model of ischemia/reperfusion (I/R). Rats were pre-injected with OA for 15 consecutive days prior to I/R operation. Subsequently, renal levels of (A) interferon (IFN)- $\gamma$, (B) interleukin (IL)-6, (C) IL-10, and (D) myeloperoxidase (MPO) were determined. Data are presented as the mean \pm standard error. ${ }^{\prime \prime} \mathrm{P}<0.05$, compared with the sham control group; ${ }^{*} \mathrm{P}<0.05$, compared with the I/R group.

$\mathbf{A}$

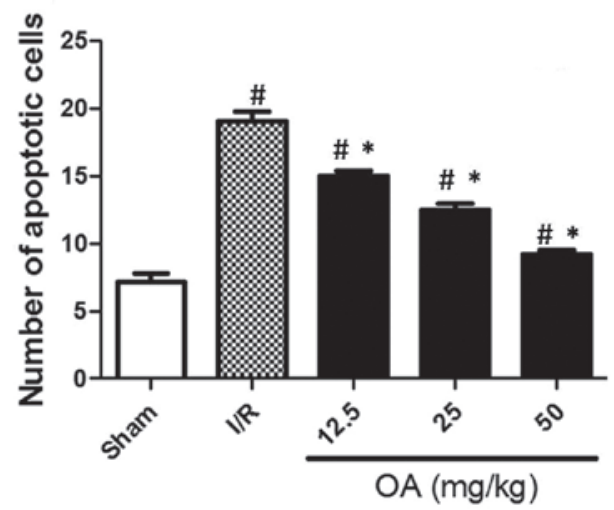

B

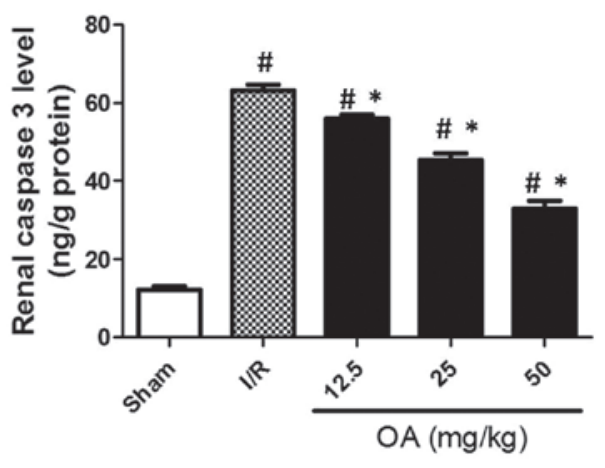

Figure 4. Effects of oleanolic acid (OA) preconditioning on apoptotic cell death in a rat model of ischemia/reperfusion (I/R). Rats were pre-injected with OA for 15 consecutive days prior to I/R operation. Subsequently, (A) renal apoptotic cell death and (B) caspase 3 content were determined. Data are presented as the mean \pm standard error. " $\mathrm{P}<0.05$, compared with the sham control group; " $\mathrm{P}<0.05$, compared with the I/R group.
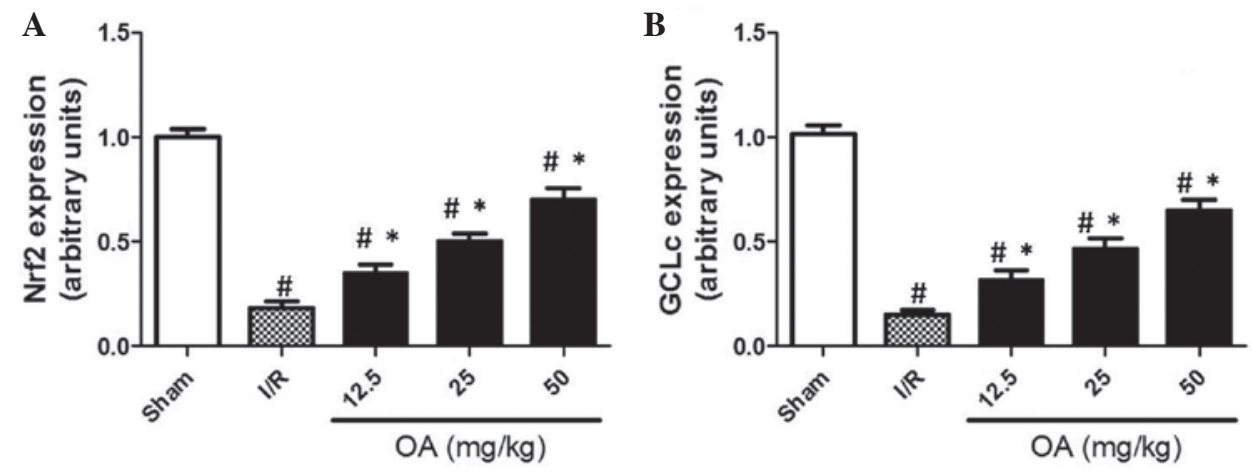

Figure 5. Effects of oleanolic acid (OA) preconditioning on nuclear factor erythroid 2-related factor 2 (Nrf2) and $\gamma$-glutamylcysteine ligase (GCLc) renal expression in a rat model of ischemia/reperfusion (I/R). Rats were pre-injected with OA for 15 consecutive days prior to I/R operation. Subsequently, renal mRNA expression of (A) Nrf2 and (B) GCLc was determined by reverse transcription-quantitative polymerase chain reaction. Data are presented as the mean \pm standard error. ${ }^{\#} \mathrm{P}<0.05$, compared with the sham control group; ${ }^{*} \mathrm{P}<0.05$, compared with the $\mathrm{I} / \mathrm{R}$ group. 

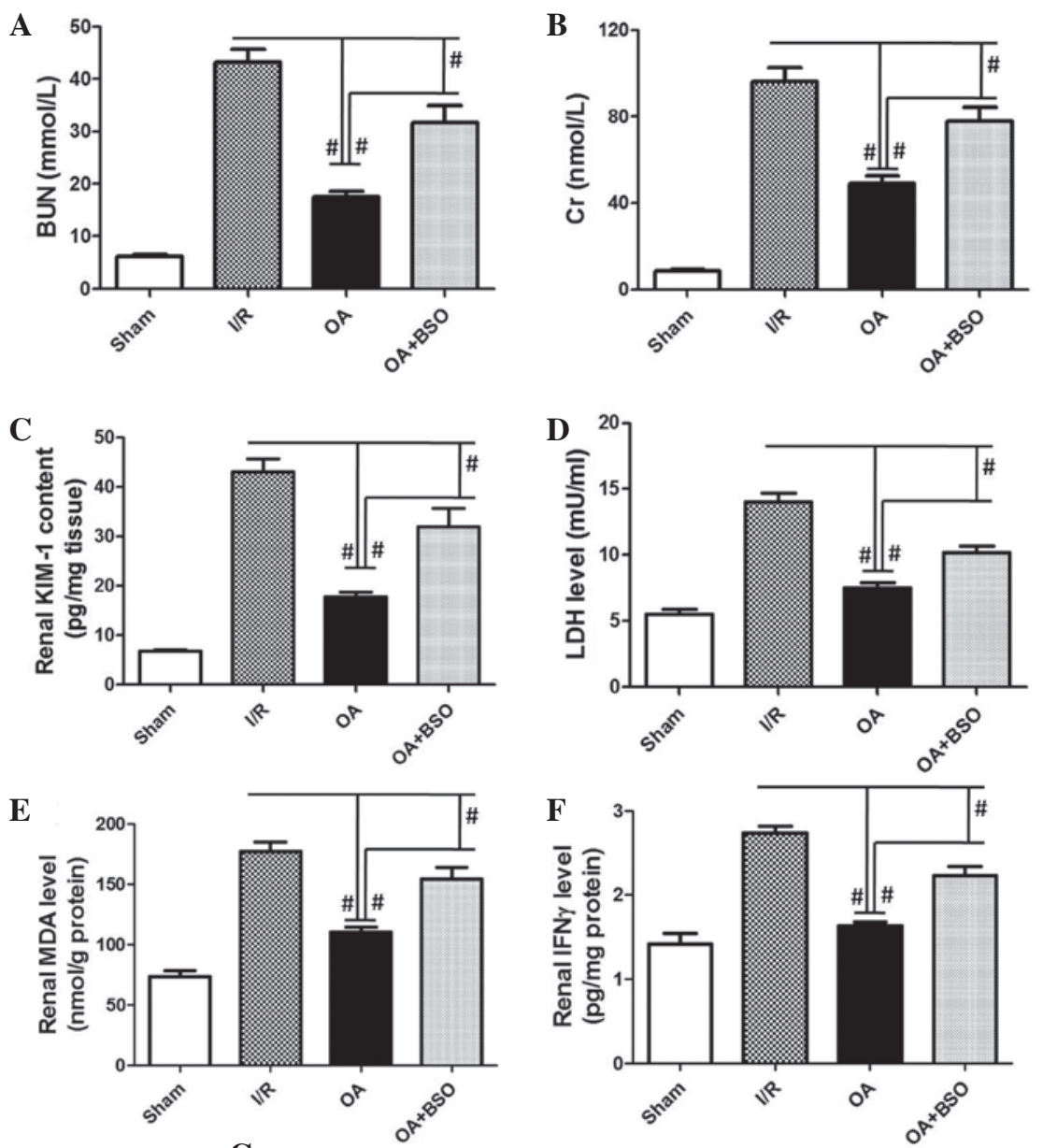

G

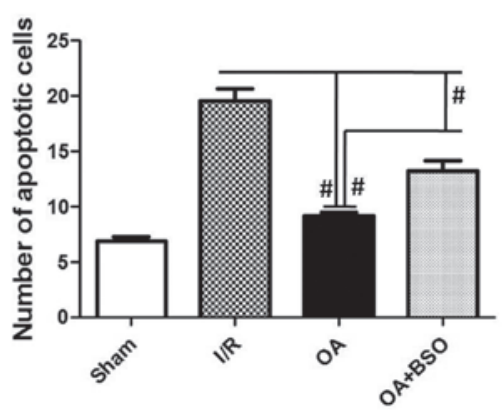

Figure 6. Role of glutathione (GSH) in the oleanolic acid (OA)-induced prevention of renal ischemia/reperfusion (I/R) injury. Rats were pre-injected with OA, with or without buthionine sulphoximine (BSO), for 15 consecutive days prior to I/R operation. Subsequently, (A) serum blood urea nitrogen (BUN), (B) serum creatinine (Cr), (C) renal kidney injury molecule-1 (KIM-1), (D) serum lactate dehydrogenase (LDH), (E) renal methane dicarboxylic aldehyde (MDA) and (F) renal interferon (IFN)- $\gamma$ levels, and (G) the number of apoptotic cells were determined. Data are presented as the mean \pm standard error. ${ }^{~} \mathrm{P}<0.05$.

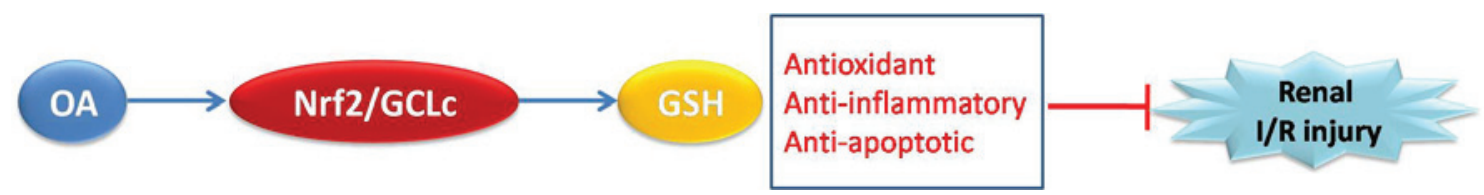

Figure 7. Mechanism underlying the protective effects of oleanolic acid (OA) preconditioning against renal ischemia/reperfusion (I/R) injury. Nrf2, nuclear factor erythroid 2-related factor 2; GCLc, $\gamma$-glutamylcysteine ligase; GSH, glutathione.

tissues. The present study examined inflammation via the detection of several cytokines and an important enzyme. As shown in Fig. 3A and B, renal levels of IFN- $\gamma$ and IL-6 were elevated in the $\mathrm{I} / \mathrm{R}$ rat group. OA preconditioning markedly prevented the increased levels of IFN- $\gamma$ and IL-6 in I/R rats (Fig. 3A and $\mathrm{B} ; \mathrm{P}=0.0045$ and $\mathrm{P}=0.0013$, respectively). Furthermore, IL-10, a cytokine that exerts protective effects against inflammatory injury (23), was decreased following I/R operation (Fig. 3C; $\mathrm{P}=0.0062)$. Conversely, $\mathrm{OA}$ preconditioning markedly prevented I/R-induced reductions in IL-10 levels (Fig. 3C). MPO is an enzyme that is stored in azurophilic granules of polymorphonuclear neutrophils and macrophages, and is released into the 
extracellular fluid in response to I/R-induced inflammation (24). The present study demonstrated that MPO levels were increased $\sim 3$-fold in the I/R group compared with in the sham control group (Fig. 3D; $\mathrm{P}=0.0012$ ). Conversely, MPO levels were inhibited by OA preconditioning in a dose-dependent manner(Fig. 3D). These results indicate that $\mathrm{I} / \mathrm{R}$ induced inflammatory injury in the rat kidneys, and the preventive effects of OA may be caused by its anti-inflammatory effects.

Anti-apoptotic effects of OA preconditioning are associated with the prevention of renal $I / R$ injury. The present study also examined the effects of OA preconditioning and I/R on apoptotic cell death in the rat kidneys. As shown in Fig. 4A, apoptotic cells were detected by TUNEL staining and presented as the number of apoptotic cells. The results demonstrated that I/R significantly increased the number of apoptotic cells in the kidneys (Fig. 4A; $\mathrm{P}=0.0006$ ). OA preconditioning notably and dose-dependently reduced the number of apoptotic cells (Fig. 4A). In addition, caspase 3, a pivotal member of the apoptotic pathway, was increased following I/R injury (Fig. 4B; P=0.0009). Conversely, OA preconditioning decreased caspase 3 content (Fig. 4B). These results indicate that the anti-apoptotic effects of OA may be associated with the prevention of I/R-induced renal injury.

Effects of OA preconditioning on Nrf2 and GCLc expression. In order to examine the mechanism underlying the protective effects of OA preconditioning against renal I/R injury in rats, the mRNA expression levels of Nrf2 and the catalytic subunit of GCLc were determined by RT-qPCR. Nrf2 is a pivotal transcription factor, which controls redox balance by regulating various antioxidant enzymes, including GCLc. GCLc is rate-limiting subunit of enzymes responsible for GSH synthesis. The present study demonstrated that $\mathrm{I} / \mathrm{R}$ resulted in a significant decrease in the expression levels of Nrf2 and GCLc (Fig. 5; $\mathrm{P}=0.0015)$. Conversely, OA preconditioning exerted a notable inhibition on I/R-induced reductions in Nrf2 and GCLc expression (Fig. 5; $\mathrm{P}=0.0021$ ). These results indicate that stabilization of Nrf2/GCLc signaling, and subsequent maintenance of the GSH pool, may be associated with the preventive effects of OA preconditioning against renal I/R injury.

Maintenance of the GSH pool is associated with the preventive effects of $O A$ preconditioning against renal I/R injury. In order to determine the role of the GSH pool in OA-induced prevention of renal I/R injury, I/R rats were pre-treated with BSO, an inhibitor of GSH synthesis. As shown in Fig. 6, OA-induced inhibition of BUN, Cr, KIM-1, LDH, MDA, IFN- $\gamma$, and apoptosis, which were increased by $\mathrm{I} / \mathrm{R}$, was significantly suppressed by $\mathrm{BSO}(\mathrm{P}=0.0002, \mathrm{P}=0.0003, \mathrm{P}=0.0001$, $\mathrm{P}=0.0002, \mathrm{P}=0.0001, \mathrm{P}=0.006, \mathrm{P}=0.001$, respectively). These results indicate that maintenance of the GSH pool is critical for OA preconditioning-induced prevention of renal I/R injury. The antioxidant activity of OA may be considered the primary capability, which contributes to the anti-inflammatory and anti-apoptotic effects of OA preconditioning (Fig. 7).

\section{Discussion}

The present study examined the effects of OA preconditioning on I/R-induced renal injury. In protocol 1, the rats were intraperitoneally injected with $12.5-50 \mathrm{mg} / \mathrm{kg}$ OA for 15 consecutive days prior to the induction of renal I/R. In protocol 2, the rats were intraperitoneally injected with $50 \mathrm{mg} / \mathrm{kg} \mathrm{OA}$, with or without $10 \mathrm{mg} / \mathrm{kg} \mathrm{BSO}$, for 15 consecutive days prior to the induction of renal $\mathrm{I} / \mathrm{R}$.

The results demonstrated that $\mathrm{OA}$ preconditioning was able to prevent I/R-induced renal injury, as evidenced by decreased serum levels of BUN, Cr and LDH, and renal levels of KIM-1, compared with the I/R group. Furthermore, OA preconditioning exhibited antioxidant effects in I/R-operated rats, as reflected by decreased MDA levels, increased SOD, CAT and GPx activities, and increased GSH content compared with the $\mathrm{I} / \mathrm{R}$ rats. OA preconditioning also exerted anti-inflammatory effects in I/R-operated rats, as shown by decreased IFN- $\gamma$, IL-6 and MPO levels, and increased IL-10 levels compared with the I/R rats. In addition, OA preconditioning exhibited anti-apoptotic effects against renal I/R injury, as reflected by decreased apoptosis and caspase 3 content. However, proapoptotic effects of OA have also been reported. For example, Wang et al (25) demonstrated that OA inhibited hepatocellular carcinoma via mitochondrial-dependent apoptosis. The discrepancy of the apoptotic effects of OA may be attributed to tissue-specific effects, or may be due to different pathological and experimental conditions. The results of the present study indicated that OA preconditioning prevented I/R-induced renal injury in rats, and the antioxidant, anti-inflammatory, and anti-apoptotic effects of OA may be associated with the prevention of renal I/R injury.

The present study also evaluated the molecular mechanism underlying the antioxidant, anti-inflammatory, and anti-apoptotic activities of OA. OA administration was able to inhibit I/R-induced decreases in Nrf2 and GCLc expression. Nrf2 is a well-known crucial regulator of cellular redox homeostasis, which has important roles in the protection against ROS damage via its ability to induce the expression of various antioxidant enzymes that detoxify ROS, and other antioxidant proteins (26). GCLc is a main target of Nrf2 transcriptional regulation (27). The regulation of GCLc by Nrf2 is associated with modulation of the GSH pool. GCLc is a subunit of the rate-limiting enzyme for de novo synthesis of GSH. GSH, which is a thiol tripeptide, is important in numerous cellular functions, including detoxifying electrophiles, scavenging ROS, maintaining the essential thiol status of proteins, and providing a reservoir for cysteine (28).

The present study examined the role of the GSH pool in the protective effects of OA preconditioning against renal $\mathrm{I} / \mathrm{R}$ injury. In the presence of $\mathrm{BSO}$, the protective effects of OA preconditioning on renal function, and the antioxidant, anti-inflammatory, and anti-apoptotic effects of OA preconditioning were significantly suppressed. The present study hypothesized that OA preconditioning stabilized Nrf2/GCLc signaling and resulted in maintenance of the GSH pool, thus defending tissues against I/R-induced renal injury. The antioxidant activity of OA may therefore be considered the major mechanism underlying the preventive effects of OA preconditioning against renal I/R injury, and may contribute to the resultant anti-inflammatory and anti-apoptotic effects.

In conclusion, the results of the present study demonstrated that OA preconditioning effectively prevented I/R-induced renal injury in rats, and this protection may be due to the 
antioxidant, anti-inflammatory, and anti-apoptotic effects of OA. OA-induced stabilization of Nrf2/GCLc signaling and maintenance of the GSH pool was shown to have a major role in the preventive effects of OA preconditioning against renal I/R injury. The present study provided initial evidence regarding the protective effects of OA preconditioning against renal I/R injury.

\section{References}

1. Gueler F, Shushakova N, Mengel M, Hueper K, Chen R, Liu X, Park JK, Haller H, Wensvoort G and Rong S: A novel therapy to attenuate acute kidney injury and ischemic allograft damage after allogenic kidney transplantation in mice. PLoS One 10: e0115709, 2015.

2. Agrawal $M$ and Swartz R: Acute renal failure. Am Fam Physician 61: 2077-2088, 2000.

3. Tuttle KR: Ischemic nephropathy. Curr Opin Nephrol Hypertens 10: 167-173, 2001.

4. Simone S, Rascio F, Castellano G, Divella C, Chieti A, Ditonno P, Battaglia M, Crovace A, Staffieri F, Oortwijn B, et al: Complement-dependent NADPH oxidase enzyme activation in renal ischemia/reperfusion injury. Free Radic Biol Med 74: 263-273, 2014

5. Niu J, Wu J, Li X and Zhang F: Association between endothelin-1/endothelin receptor $\mathrm{A}$ and inflammation in mouse kidneys following acute ischemia/reperfusion. Mol Med Rep 11: 3981-3987, 2015

6. Wang L, Liu X, Chen H, Chen Z, Weng X, Qiu T and Liu L: Effect of picroside II on apoptosis induced by renal ischemia/reperfusion injury in rats. Exp Ther Med 9: 817-822, 2015.

7. Dong B, Zhou H, Han C, Yao J, Xu L, Zhang M, Fu Y and Xia Q: Ischemia/reperfusion-induced CHOP expression promotes apoptosis and impairs renal function recovery: The role of acidosis and GPR4. PLoS One 9: e110944, 2014.

8. Cruthirds DL, Novak L, Akhi KM, Sanders PW, Thompson JA and MacMillan-Crow LA: Mitochondrial targets of oxidative stress during renal ischemia/reperfusion. Arch Biochem Biophys 412: 27-33, 2003.

9. Yang B, Jain S, Pawluczyk IZ, Imtiaz S, Bowley L, Ashra SY and Nicholson ML: Inflammation and caspase activation in long-term renal ischemia/reperfusion injury and immunosuppression in rats. Kidney Int 68: 2050-2067, 2005.

10. Wu HH, Hsiao TY, Chien CT and Lai MK: Ischemic conditioning by short periods of reperfusion attenuates renal ischemia/reperfusion induced apoptosis and autophagy in the rat. J Biomed Sci 16: 19, 2009.

11. El Morsy EM, Ahmed MA and Ahmed AA: Attenuation of renal ischemia/reperfusion injury by açaí extract preconditioning in a rat model. Life Sci 123: 35-42, 2015.

12. Ilhan H, Eroglu M, Inal V, Eyi YE, Arziman I, Yildirim AO, Tansel A, Uzun G and Yamanel L: Hyperbaric oxygen therapy alleviates oxidative stress and tissue injury in renal ischemia/reperfusion injury in rats. Ren Fail 34: 1305-1308, 2012.
13. Chen H, Xing B, Liu X, Zhan B, Zhou J, Zhu H and Chen Z: Ozone oxidative preconditioning inhibits inflammation and apoptosis in a rat model of renal ischemia/reperfusion injury. Eur J Pharmacol 581: 306-314, 2008.

14. Pérez-Camino MC and Cert A: Quantitative determination of hydroxy pentacyclic triterpene acids in vegetable oils. J Agric Food Chem 47: 1558-1562, 1999.

15. Wang X, Ye XL, Liu R, Chen HL, Bai H, Liang X, Zhang XD, Wang Z, Li WL and Hai CX: Antioxidant activities of oleanolic acid in vitro: Possible role of Nrf2 and MAP kinases. Chem Biol Interact 184: 328-337, 2010

16. Liu J, Wang X, Liu R, Liu Y, Zhang T, Fu H and Hai C: Oleanolic acid co-administration alleviates ethanol-induced hepatic injury via Nrf-2 and ethanol-metabolizing modulating in rats. Chem Biol Interact 221: 88-98, 2014.

17. Nkeh-Chungag BN, Oyedeji OO, Oyedeji AO and Ndebia EJ: Anti-inflammatory and membrane-stabilizing properties of two semisynthetic derivatives of oleanolic acid. Inflammation 38: 61-69, 2015

18. Bischoff A, Bucher M, Gekle M and Sauvant C: Differential effect of COX1 and COX2 inhibitors on renal outcomes following ischemic acute kidney injury. Am J Nephrol 40: 1-11, 2014.

19. Wills ED: Mechanisms of lipid peroxide formation in animal tissues. Biochem J 99: 667-676, 1966.

20. Wang X, Hai CX, Liang X, Yu SX, Zhang W and Li YL: The protective effects of Acanthopanax senticosus Harms aqueous extracts against oxidative stress: Role of Nrf2 and antioxidant enzymes. J Ethnopharmacol 127: 424-432, 2010.

21. Grimholt RM, Urdal P, Klingenberg O and Piehler AP: Rapid and reliable detection of $\alpha$-globin copy number variations by quantitative real-time PCR. BMC Hematol 14: 4, 2014.

22. Han WK, Bailly V, Abichandani R, Thadhani R and Bonventre JV: Kidney Injury Molecule-1 (KIM-1): A novel biomarker for human renal proximal tubule injury. Kidney Int 62: 237-244, 2002.

23. Vázquez-Frias R, Gutiérrez-Reyes G, Urbán-Reyes $M$, Velázquez-Guadarrama N, Fortoul-van der Goes TI, Reyes-López A and Consuelo-Sánchez A: Proinflammatory and anti-inflammatory cytokine profile in pediatric patients with irritable bowel syndrome. Rev Gastroenterol Mex 80: 6-12, 2015.

24. Loria V, Dato I, Graziani F and Biasucci LM: Myeloperoxidase: A new biomarker of inflammation in ischemic heart disease and acute coronary syndromes. Mediators Inflamm 2008: 135625, 2008.

25. Wang X, Bai H, Zhang X, Liu J, Cao P, Liao N, Zhang W, Wang Z and Hai C: Inhibitory effect of oleanolic acid on hepatocellular carcinoma via ERK-p53-mediated cell cycle arrest and mitochondrial-dependent apoptosis. Carcinogenesis 34: 1323-1330, 2013.

26. Kwak MK, Wakabayashi N, Itoh K, Motohashi H, Yamamoto M and Kensler TW: Modulation of gene expression by cancer chemopreventive dithiolethiones through the Keap1-Nrf2 pathway. Identification of novel gene clusters for cell survival. J Biol Chem 278: 8135-8145, 2003.

27. Lu YF, Liu J, Wu KC, Qu Q, Fan F and Klaassen CD: Overexpression of Nrf2 protects against microcystin-induced hepatotoxicity in mice. PLoS One 9: e93013, 2014.

28. Arrick BA and Nathan CF: Glutathione metabolism as a determinant of therapeutic efficacy: A review. Cancer Res 44: 4224-4232, 1984. 